\title{
Histological, serological and virulence studies on rainbow trout experimentally infected with recombinant infectious hematopoietic necrosis viruses
}

\author{
Alejandro Romero ${ }^{1}$, Antonio Figueras ${ }^{1}$, Carolina Tafalla $^{1}$, Maria-Isabel Thoulouze $^{2}$, \\ Michel Bremont ${ }^{2}$, Beatriz Novoa ${ }^{1, *}$ \\ ${ }^{1}$ Instituto de Investigaciones Marinas, Consejo Superior de Investigaciones Cientificas (CSIC), Eduardo Cabello 6, \\ 36208 Vigo, Spain \\ ${ }^{2}$ Unité de Virologie et Immunologie Moléculaires, Centre de Recherche de Jouy-en-Josas, Institut National de la Recherche \\ Agronomique (INRA), 78352 Jouy-en-Josas Cedex, France
}

\begin{abstract}
Several recombinant infectious hematopoietic necrosis viruses (IHNV) were produced by reverse genetics and their pathogenicity in trout was evaluated and compared to that of the wild type (wt) viruses IHNV and viral haemorrhagic septicemia virus (VHSV). Recombinant IHNVs used in this study were: rIHNV, identical to the wtIHNV; rIHNV-Gvhsv, a recombinant virus expressing the VHSV G gene instead of the IHNV G gene; rIHNV-Gmut, which possesses 2 targeted mutations in the glycoprotein; and rIHNVmut-Gmut, which is similar to the rIHNV-Gmut, but exhibits additional mutations along the genome. Results obtained in experimental infections showed that the rIHNV and rIHNV-Gmut were the most virulent recombinant viruses. Severity of the lesions induced by the different recombinant viruses was in agreement with mortality data. The kidney and the liver were the organs most affected by the most pathogenic viruses, and the lesions observed resembled those produced by wtIHNV. The introduction of mutations did not alter the tissue tropism of the virus. The recombinant viruses were able to replicate in fish, as shown by immunoperoxidase assay and RTPCR. Antibodies against IHNV were detected in the fish inoculated with IHNV, rIHNV, rIHNV-Gmut and rIHNVmut-Gmut, and antibodies against VHSV were also found in fish infected with rIHNVGvhsv. Finally, antibody production was highest in fish infected with the rIHNVmut-Gmut even though this virus was the least virulent.
\end{abstract}

KEY WORDS: Rhabdovirus - Infectious hematopoietic necrosis virus · IHNV • Viral hemorrhagic septicemia virus $\cdot \mathrm{VHSV} \cdot$ Oncorhynchus mykiss $\cdot$ Reverse genetics $\cdot$ Recombinant virus

Resale or republication not permitted without written consent of the publisher

\section{INTRODUCTION}

Infectious hematopoietic necrosis virus (IHNV) and viral haemorrhagic septicemia virus (VHSV) are 2 rhabdoviruses, probably the most important viral pathogens in fish, responsible for high mortalities in farmed salmonids (Bootland \& Leong 1999, Smail 1999). Both are the cause of notifiable diseases that occur in the European Union, and are classified as such by the OIE (Office International des Epizooties). As with all rhabdoviruses, the genomes of IHNV and VHSV consist of single-stranded negative-sense RNA which has been entirely sequenced (Schütze et al.
1995, Schütze et al. 1999). Their genomes code for 5 structural proteins: a nucleoprotein $(\mathrm{N})$, a polymeraseassociated protein $(\mathrm{P})$, a matrix protein $(\mathrm{M})$, a surface glycoprotein (G) responsible for immunogenicity (McAllister \& Wagner 1975) and an RNA-dependent RNA polymerase (L). An additional gene, only present in some fish rhabdoviruses, located between the $\mathrm{G}$ and $\mathrm{L}$ genes, encodes a non-structural protein (NV), whose role is unknown (Kurath \& Leong 1985) but appears to be linked to viral pathogenicity (Thoulouze et al. 2004). Since no effective therapies are available for fish viral diseases, vaccination is a potential tool to control these deleterious viruses. 
A potentially powerful approach in vaccine development for single-stranded RNA viruses is based on the reverse genetics strategy. Reverse genetics, developed for single-stranded RNA viruses, allows RNA genome manipulation, which may help in investigations of the functions of viral protein and genetic elements. This technique enables the functional replacement of viral proteins and the expression of additional proteins by the insertion of new genes into the viral genome. Viruses may be attenuated by the introduction of targeted mutations in their RNA genomes, which could be a useful tool for vaccine development. The reverse genetics technology used here is based on the recovery of recombinant viruses from T7 RNA polymerase vaccinia-infected cells transfected with a T7-driven expression plasmid coding for the full-length antigenomic cDNA together with T7-driven expression plasmid coding for the nucleoprotein $(\mathrm{N})$, the phosphoprotein (P) and the RNA polymerase (L) (Biacchesi et al. 2000). This technique has been developed for different rhabdoviruses such as rabies virus (RV) (Schnell et al. 1994, Ito et al. 2001), vesicular stomatitis virus (VSV) (Lawson et al. 1995, Whelan et al. 1995), snakehead rhabdovirus (SHRV) (Johnson et al. 2000) and IHNV (Biacchesi et al. 2000).

The introduction of targeted mutations in the viral genome is particularly practicable in the gene encoding the $\mathrm{G}$ protein, which has been shown to be the main elicitor of the fish immune response against these viruses (Engelking \& Leong 1989a,b), and to be the target of neutralizing antibodies (Huang et al. 1996). Kim et al. (1994) generated 5 neutralization-resistant variants of IHNV, using a monoclonal antibody (MAb) directed against IHNV-G gene. In vivo analysis of virus-induced pathogenesis revealed that all the variants had altered virulence and exhibited differences in tissue tropism in rainbow trout (Kim et al. 1994). One of them, RB-1, was described to be particularly attenuated, and the loss of virulence was correlated with either or both amino acid changes at position 78 and 218 of the $\mathrm{G}$ protein. In the present study, in order to determine whether these 2 point mutations detected in the $\mathrm{G}$ gene were indeed the molecular basis of virus attenuation, reverse genetics was used to create 2 recombinant generations, and specific mutations were introduced in the $\mathrm{G}$ gene (amino acid position 78 and 218 ) to induce changes in the amino acid of the G protein, making it identical to that of RB-1 (rIHNV-Gmut). A second virus was also generated (rIHNVmut-Gmut) containing 5 incidental mutations located in the $\mathrm{N}$ and the $\mathrm{M}$ gene in addition to the 2 targeted mutations (G78 and 218) (see Fig. 1). As a first step to determine if any of these recombinant viruses could be used as a vaccine, we studied their pathogenicity, and the level of antibody production that each of them elicited, in experimentally infected rainbow trout.

\section{MATERIALS AND METHODS}

Fish. Rainbow trout Oncorhynchus mykiss were obtained from a commercial fish farm (Juan Queiro S.A., Spain). Fish were acclimatised to laboratory conditions for $2 \mathrm{wk}$. Experiments were performed in $50 \mathrm{l}$ closed circuit tanks, with aeration, at $14^{\circ} \mathrm{C}$. Fish were fed daily with dry pellet food.

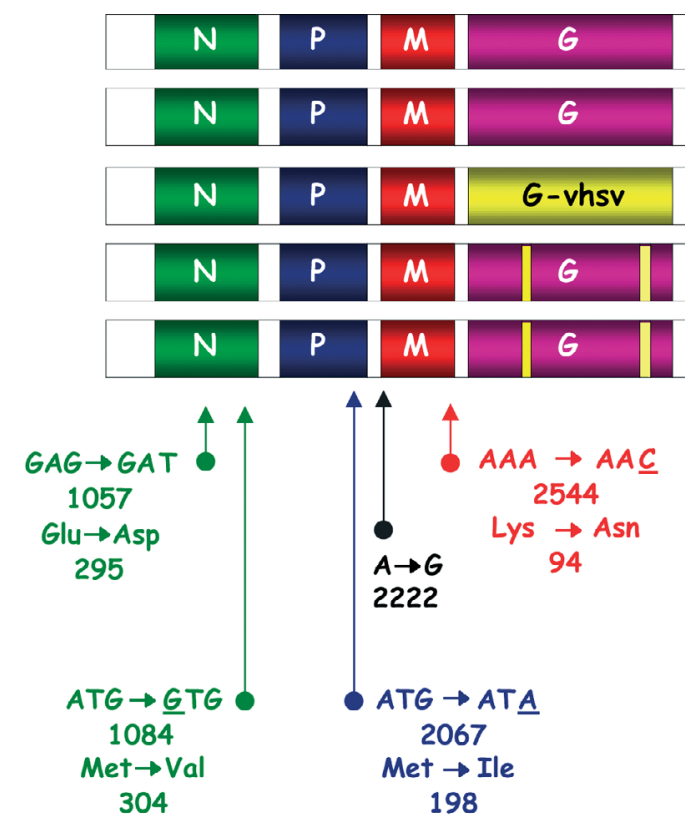

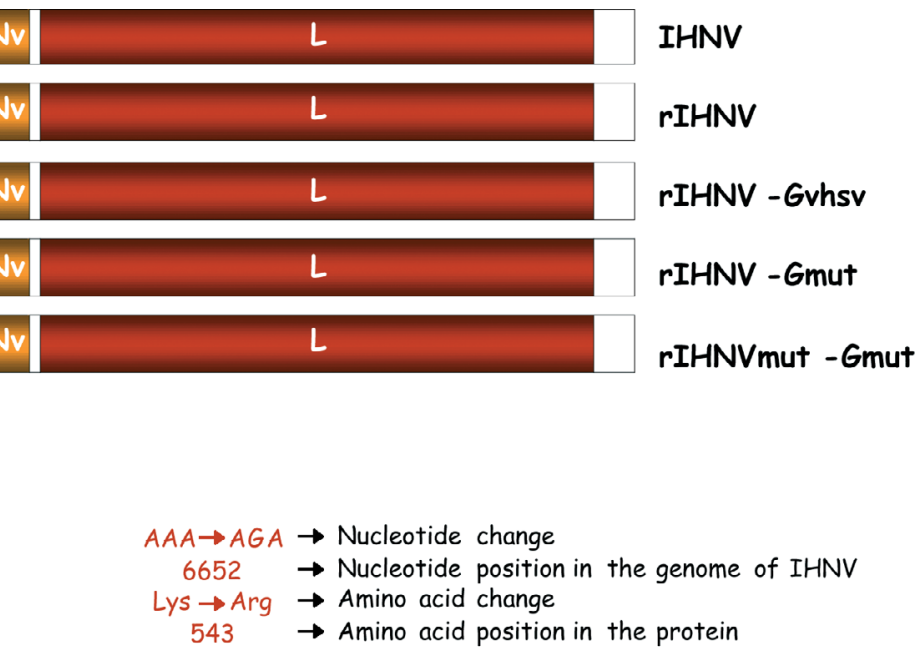

Fig. 1. Scheme representing the genome of the different recombinant viruses used in this study. The localization of the 5 incidental mutations on the genome of the recombinant virus rIHNVmutGmut is shown in detail 
Viruses and cell line. Recombinant viruses used in this study (rIHNV, rIHNV-Gvhsv, rIHNV-Gmut and rIHNVmut-Gmut) were produced in our laboratory and were described by Biacchesi et al. $(2000,2002)$. Fig. 1 shows a detailed localization of mutations of the rIHNVmut-Gmut out of the G gene. The wild type (wt) virus strains used in this study were French isolates IHNV 32/87 and VHSV 07-71 (Biacchesi et al. 2000).

Viruses were replicated in the fish cell line epithelioma papulosum cyprini (EPC) derived from common carp Cyprinus carpio (Tomasec \& Fijan 1971). EPC cells were cultured in Eagle's minimum essential medium (MEM, Gibco) supplemented with foetal bovine serum (FBS), penicillin $\left(100 \mathrm{IU} \mathrm{ml}^{-1}\right)$ and streptomycin $(100 \mu \mathrm{g}$ $\mathrm{ml}^{-1}$ ) and buffered with $7.5 \%$ sodium bicarbonate, and incubated at $20^{\circ} \mathrm{C}$.

Experimental infections. Infection experiments were carried out to estimate the virulence of the different IHNV recombinants through bath challenge and intraperitoneal injection.

In the experimental infections by bath immersion, groups of 30 rainbow trout ( 4 to $7 \mathrm{~cm}, 5$ to $7 \mathrm{~g}$ mean,) were exposed for $1 \mathrm{~h}$ in $2.5 \mathrm{l}$ to a virus preparation containing 2000 plaque forming units per $\mathrm{ml}\left(\mathrm{pfu} \mathrm{ml}{ }^{-1}\right.$ ) of rIHNV, rIHNV-Gmut, rIHNVmut-Gmut, rIHNV-Gvhsv and wtIHNV. The water volume in tanks was then restored to $50 \mathrm{l}$ and the fish were kept in a closed circuit water system.

In the challenge experiments performed by intraperitoneal injection, groups of 25 rainbow trout ( 4 to $7 \mathrm{~cm}, 5$ to $7 \mathrm{~g}$ mean) were injected intraperitoneally with $100 \mu \mathrm{l}$ of a $2 \times 10^{6} \mathrm{pfu} \mathrm{ml}^{-1}$ dilution of the different recombinants or wt viruses (IHNV and VHSV). Fish were also kept in 501 tanks with a closed circuit water system.

In all cases, mortalities were recorded daily for $24 \mathrm{~d}$.

Histology. Two different experiments were conducted to study histological lesions induced by the recombinants of IHNV. In both cases, rainbow trout were distributed into 30 -fish groups ( 4 to $7 \mathrm{~cm}, 5$ to $7 \mathrm{~g}$ mean) in $50 \mathrm{l}$ tanks. Each group was intraperitoneally injected with $100 \mu \mathrm{l}$ of each of the IHNV recombinants or with wtIHNV $\left(5 \times 10^{6} \mathrm{pfu} \mathrm{ml}^{-1}\right)$. A control group was injected with culture medium.

In the first trial, after $17 \mathrm{~d}$ of infection, 4 survivor fish from each group were sampled and the muscle, kidney, liver, spleen and brain processed for histology. In the second trial, the evolution of the histological lesions was evaluated on 4 fish from each group at Days 4, 8 and 14 post-infection (p.i.). The same organs were sampled.

In all cases, transversal sections, approximately $5 \mathrm{~mm}$ thick, were excised from the organs and fixed in $4 \%$ buffered formalin, embedded in paraffin and stained with hematoxylin and eosin.
A grading system for the most frequent observed lesions in the kidney and liver was established. Haemorrhages and necrosis were classified into 4 intensity levels ranging from 0 (almost no erythrocytes and no necrotic lesions were detected in the organ) to 3 (high numbers of erythrocytes and large necrotic areas were observed). In the liver, the disappearance of the hepatic cell cord structure and the cytoplasmatic vacuolization were classified in 3 different intensity levels, from 0 (control fish) to 2 (complete disappearance of the cell cord structure and cytoplasmatic vacuolization extended in all the organ).

Viral replication. The viral replication was studied by an immunoperoxidase assay performed on cell cultures that had been inoculated with homogenates obtained from the sampled organs, as well as by a semi-nested reverse transcriptase-polymerase chain reaction (RT-PCR).

We used the immunoperoxidase assay protocol described by Aranguren et al. (2002) with some modifications. Briefly, kidney, liver, spleen and brain from trout intraperitoneally injected $\left(100 \mu \mathrm{l}, 5 \times 10^{6} \mathrm{pfu} \mathrm{ml}^{-1}\right)$ with the different IHNV recombinants or wtIHNV as well as from control fish, were obtained at Days 3, 7 and 15 p.i. ( $\mathrm{n}=4$ in each group). Organs were homogenised in culture medium (MEM) supplemented with penicillin (200 IU ml $\left.{ }^{-1}\right)$, streptomycin $\left(200 \mu \mathrm{g} \mathrm{ml}^{-1}\right)$, gentamicin $\left(20 \mu \mathrm{g} \mathrm{ml}^{-1}\right)$ and fungizone $\left(2 \mu \mathrm{g} \mathrm{ml}^{-1}\right)$ with a manual potter and the homogenates inoculated into EPC cells grown in 96 well plates. After $24 \mathrm{~h}$ of incubation at $14^{\circ} \mathrm{C}$, the virus was detected by immunoperoxidase directly on cells, using a monoclonal antibody directed against IHNV-P (1:1000) (supplied by M. Bremont) and an anti-mouse Ig G peroxidase conjugate (1:500). Activated aminoethylcarbazole (AEC-101 Staining Kit, Sigma) was used for staining.

Detection of the recombinant viruses in the different organs was done using a semi-nested RT-PCR. Groups of trout $(\mathrm{n}=15)$ were intraperitoneally injected with $100 \mu \mathrm{l}$ of either the wild type IHNV or the different recombinants $\left(2 \times 10^{6} \mathrm{pfu} \mathrm{ml}^{-1}\right)$. After 1 and $3 \mathrm{~d}$ postinjection, 3 fish from each group were sampled and the kidney, liver, spleen and gills removed. RNA extraction was performed using Trizol Reagent (Invitrogen) following the manufacturer indications. The reverse transcription and the posterior semi-nested PCR were performed following the procedure described by Miller et al. (1998)

Antibody production. The ability of the different recombinant IHNVs to induce antibody production in trout was determined as follows. Rainbow trout (15 to $20 \mathrm{~cm}, 20$ to $25 \mathrm{~g}$ mean) were intraperitoneally injected with $250 \mu \mathrm{l}$ of a solution containing $10^{3} \mathrm{pfu} \mathrm{ml}^{-1}$ of each virus. Blood was drawn from the caudal vein after 4 , 7 and $10 \mathrm{wk}$ of exposure to the viruses and clotted 
overnight. Plasma was obtained by centrifugation for 15 min at $500 \times g$ in a refrigerated centrifuge. Plasma was stored at $-20^{\circ} \mathrm{C}$ until antibody production was quantified by an enzyme-linked immunosorbent assay (ELISA).

For the ELISA, 96-well plates were coated with IHNV $\left(2 \times 10^{6} \mathrm{pfu} \mathrm{ml}^{-1}, 50 \mu \mathrm{l} \mathrm{well}^{-1}\right)$ diluted in coating buffer $\left(\mathrm{Na}_{2} \mathrm{CO}_{3} 0.0015 \mathrm{M}, \mathrm{NaHCO}_{3} 0.03 \mathrm{M}, \mathrm{pH}\right.$ 9.6) and left overnight at $4{ }^{\circ} \mathrm{C}$. All unbound sites were then blocked with phosphate buffered saline Tween-20 (PBS + 1\% Tween-20) with $3 \%$ bovine serum albumin (BSA) for $1 \mathrm{~h}$ at $37^{\circ} \mathrm{C}$. Plasma dilutions were performed in PBS-T $+1 \%$ BSA and added in duplicate to precoated and blocked ELISA plates. After $1 \mathrm{~h}$ of incubation at $37^{\circ} \mathrm{C}$, plates were washed twice with PBS-T and incubated with polyclonal anti-trout Ig (1G7) (Sánchez et al. 1991) diluted 1:500 in PBS-T for $2 \mathrm{~h}$ at room temperature, and revealed by a further $1 \mathrm{~h}$ incubation with a peroxidase conjugated anti-IgG antibody diluted 1:1000 in PBS. After 2 washes in TBS-T, the enzyme substrate, orthophenylendiamine (OPD) (Sigma) was added and the reaction was stopped 20 min later with the addition of $3 \mathrm{M}$ sulphuric acid. The reaction intensity was measured by reading the optical density at $492 \mathrm{~nm}$ and the antibody titer was calculated, taking a sample as standard, by the method described by Shapiro et al. (1997). In the case of samples taken from fish inoculated with rIHNV-Gvhsv, an additional ELISA was performed to quantify antibodies against VHSV using the same protocol.

\section{RESULTS}

\section{Virulence of IHNV recombinant viruses}

All the recombinant viruses described in this work (rIHNV, rIHNV-Gvhsv, rIHNV-Gmut and rIHNVmutGmut) were compared to wtIHNV for their pathogenicity in juvenile rainbow trout, by analyzing the disease symptoms and the mortalities induced by each virus. Thus, samples of 30 rainbow trout (4 to $7 \mathrm{~cm}$ mean) were infected with each of the IHNV recombinants either by the bath immersion route $\left(10^{3} \mathrm{PFU} \mathrm{ml} \mathrm{m}^{-1}\right)$ or by intraperitoneal injection $\left(10^{6} \mathrm{PFU}\right.$ $\mathrm{ml}^{-1}$ ). As a positive control, wtIHNV was included and a negative control group was injected with culture medium. Symptoms (nature and intensity) and mortalities were recorded every day for $24 \mathrm{~d}$ after virus exposure.

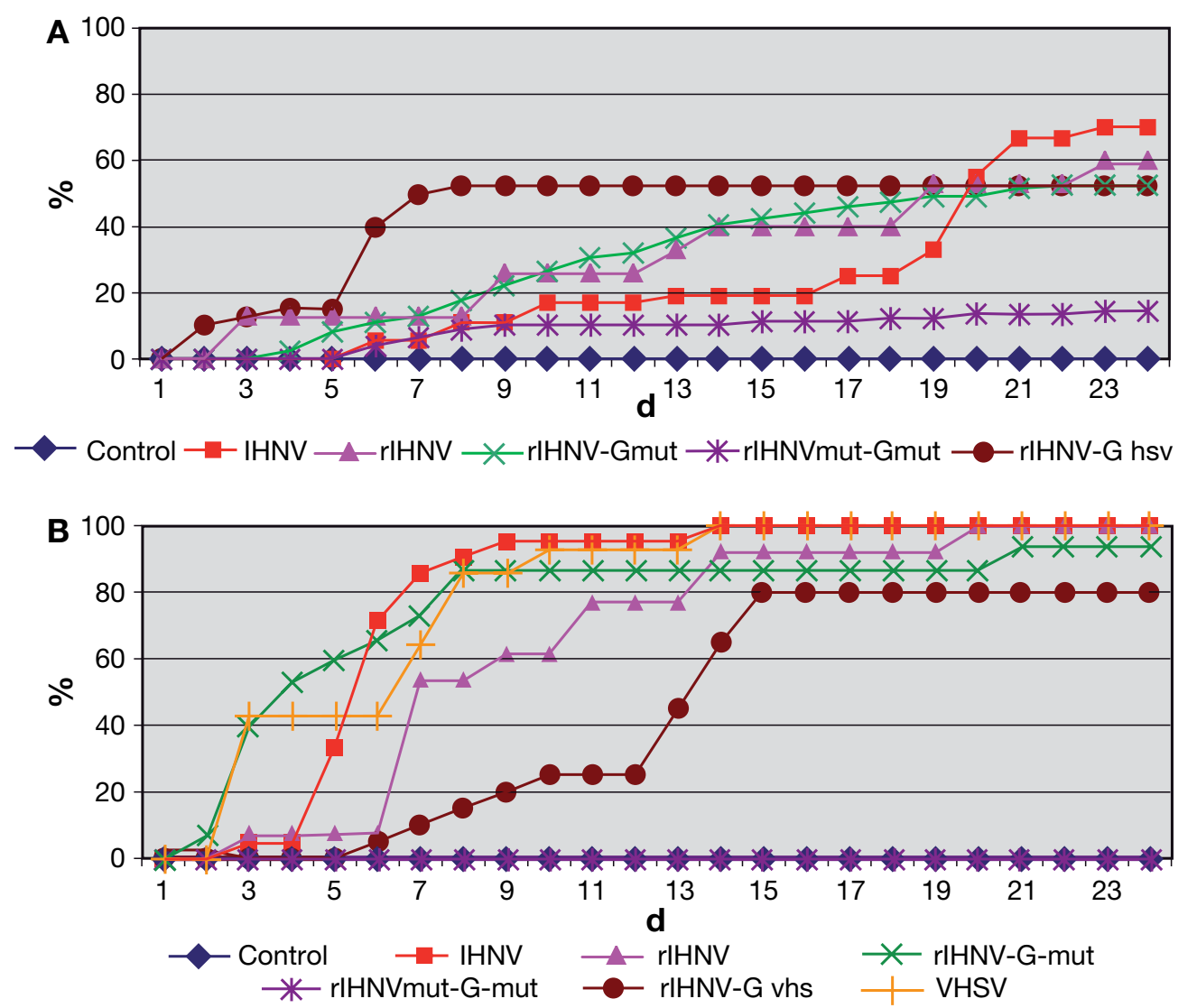

Fig. 2. Oncorhynchus mykiss. Mortalities following infection with IHNV and the different recombinant IHNVs. Control (uninfected) fish were mock-infected with culture medium. (A) Bath (30 fish, 4 to $7 \mathrm{~cm}$ ). (B) Intraperitoneal injection (25 fish, 4 to $7 \mathrm{~cm}$ ) 
In bath-infected fish, virus-induced deaths began $4 \mathrm{~d}$ after infection. Mortality increased slowly during a $16 \mathrm{~d}$ period and reached a maximum rate after $6 \mathrm{~d}$. Cumulative mortalities of fish infected through bath challenge with wtIHNV, rIHNV, rIHNV-Gmut and rIHNV-Gvhsv were between $66 \%$ and $52 \%$ after $24 \mathrm{~d}$ (Fig. 2A). For recombinant rIHNVmut-Gmut, mortalities were delayed by $2 \mathrm{~d}$ in comparison with those caused by the rIHNV-Gmut, and death occurred more slowly and at a much lower rate, reaching $14 \%$ by Day 24 p.i. No mortality was recorded in control mock-infected fish.

In fish infected by intraperitoneal injection (Fig. 2B), the first mortalities occurred $2 \mathrm{~d}$ p.i. Wild type viruses (IHNV and VHSV) and the rIHNV virus took $14 \mathrm{~d}$ and $20 \mathrm{~d}$ respectively to reach the maximum mortality $(100 \%)$. rIHNV-Gvhsv induced a delayed mortality but cumulative mortalities finally reached a plateau at $80 \%$ by Day 16 p.i. Interestingly, rIHNV-Gmut also induced a high mortality rate, reaching $93 \%$ after $21 \mathrm{~d}$. However, the rIHNVmut-Gmut, with 7 mutations in the viral genome, did not induce mortalities.

For rIHNV, rIHNV-Gmut and rIHNV-Gvhsv, infected moribund fish exhibited external symptoms similar to those produced by wtIHNV (Fig. 3): dark coloration, distended visceral cavity, exophthalmia and pale gills. Some fish discharged opaque faecal casts and had petechial haemorrhages at the base of the fins and abdomen. After $8 \mathrm{~d}$, liver, spleen and kidney from moribund fish were pale, the stomach was filled with a milky fluid and the intestine contained a watery, yellowish fluid. No external symptoms were observed in fish treated with rIHNVmut-Gmut.

Altogether, these data indicated that not only rIHNV, rIHNV-Gvhsv, but also rIHNV-Gmut, are highly pathogenic for rainbow trout, inducing the same symptoms as wtIHNV, whereas rIHNVmut-Gmut exhibited an attenuated virulence.

\section{Histopathology}

A histological analysis was performed to compare the lesions associated with each recombinant virus in different tissues. The severities of the lesions (Table 1) were recorded using the grading system established for the most frequent observed lesions in the kidney and liver (see 'Materials and methods').

Table 1. Intensity of the histological lesions induced by the different recombinant viruses on Days 4, 8 and 14 . (-) No lesions. $( \pm)$ Some morphological changes and slight lesions. (+) Appearance of lesions with different intensities from $(+)$ low intensity, to $(++++)$ high intensity

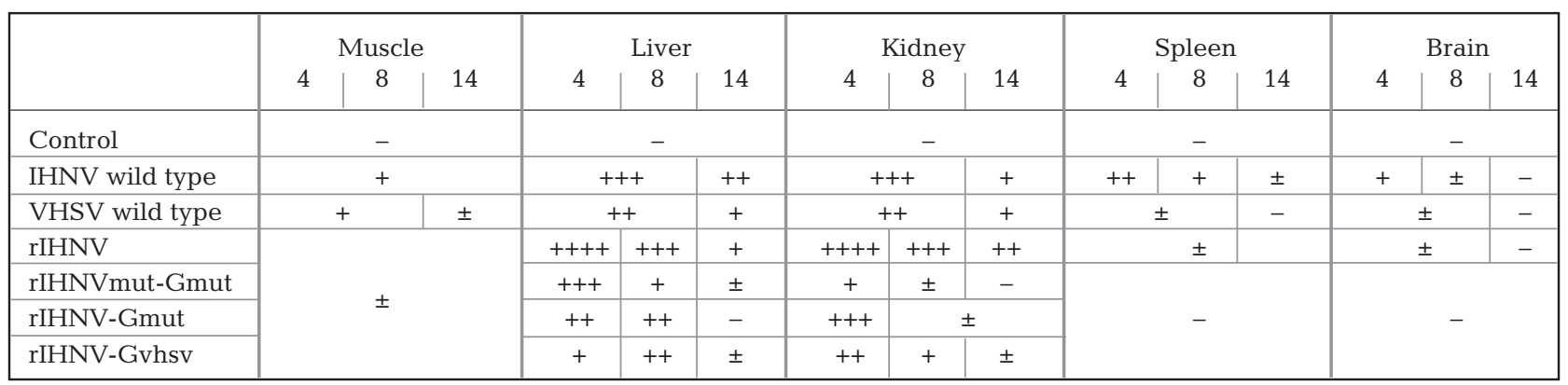
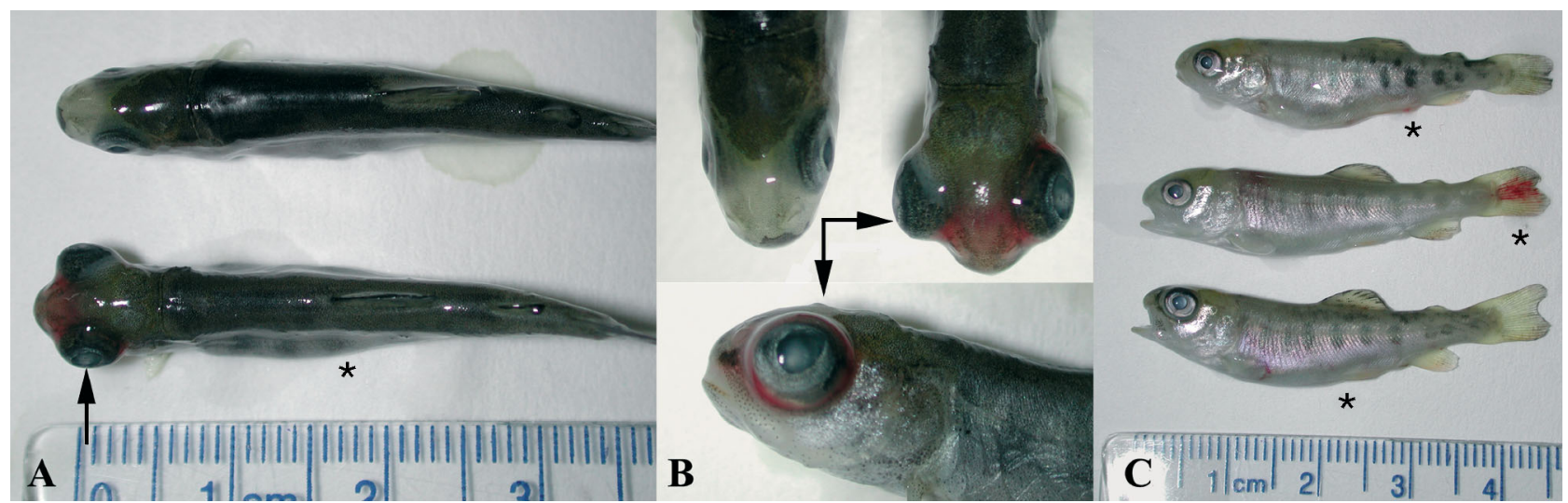

Fig. 3. Oncorhynchus mykiss. Clinical symptoms observed for wtIHNV and recombinant viruses (rIHNV, rIHNV-Gmut and rIHNV-Gvhsv) in experimentally infected fish. Arrows in (A) and (B) show exophthalmia and ocular haemorrhages. (*) indicates a distended abdomen in $(\mathrm{A})$, and petechial haemorrhages at the base of the fins and vent in $(\mathrm{C})$ 

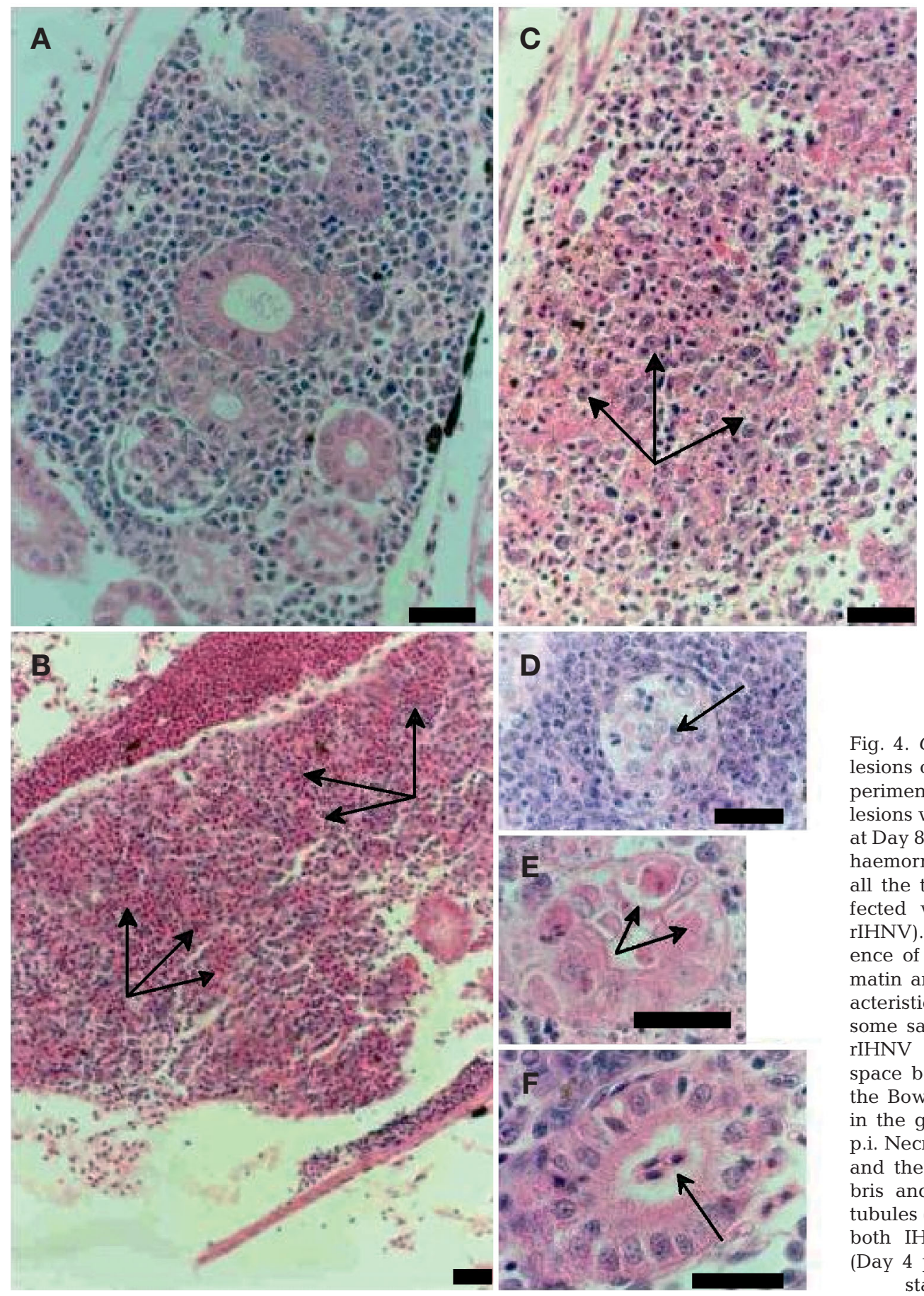

Fig. 4. Oncorhynchus mykiss. Main lesions observed in kidney from experimentally infected fish. (A) No lesions were observed in control fish at Day 8 p.i. (B) Arrows show intense haemorrhages extended throughout all the tissue at Day 4 p.i. (fish infected with IHNV wild type and rIHNV). (C) Arrows show the presence of cells with condensed chromatin and prominent nucleoli characteristic of the necrotic areas. (D) In some samples IHNV wild type and rIHNV induced clogging of the space between the glomerulus and the Bowman's capsule and necrosis in the glomerulus (arrow) at Day 4 p.i. Necrosis in tubules (arrows in E) and the appearance of cellular debris and erythrocytes in the renal tubules (arrow in F) were frequent in both IHNV and rIHNV infections (Day 4 p.i). Hematoxylin and eosin stain. Scale bars $=20 \mu \mathrm{m}$

For all the recombinant viruses included in this study (rIHNV, rIHNV-Gvhsv, rIHNV-Gmut and rIHNVmutGmut), the kidney and the liver were the main target organs, as already described for wtIHNV. For wtIHNV, the kidney of rainbow trout 4 and $8 \mathrm{~d}$ after infection showed intense haemorrhages throughout all the tissue (Fig. 4B), as well as necrotic areas with cellular debris and cells with condensed chromatin and prominent nucleoli (Fig. 4C). Moreover, wtIHNV induced clogging of the space between the glomerulus and the Bowman's capsule (Fig. 4D) and necrosis and tubular degeneration (Fig. 4E). Cellular debris and erythrocytes in the renal tubules were frequent (Fig. 4F). The liver exhibited a total or partial disappearance of 

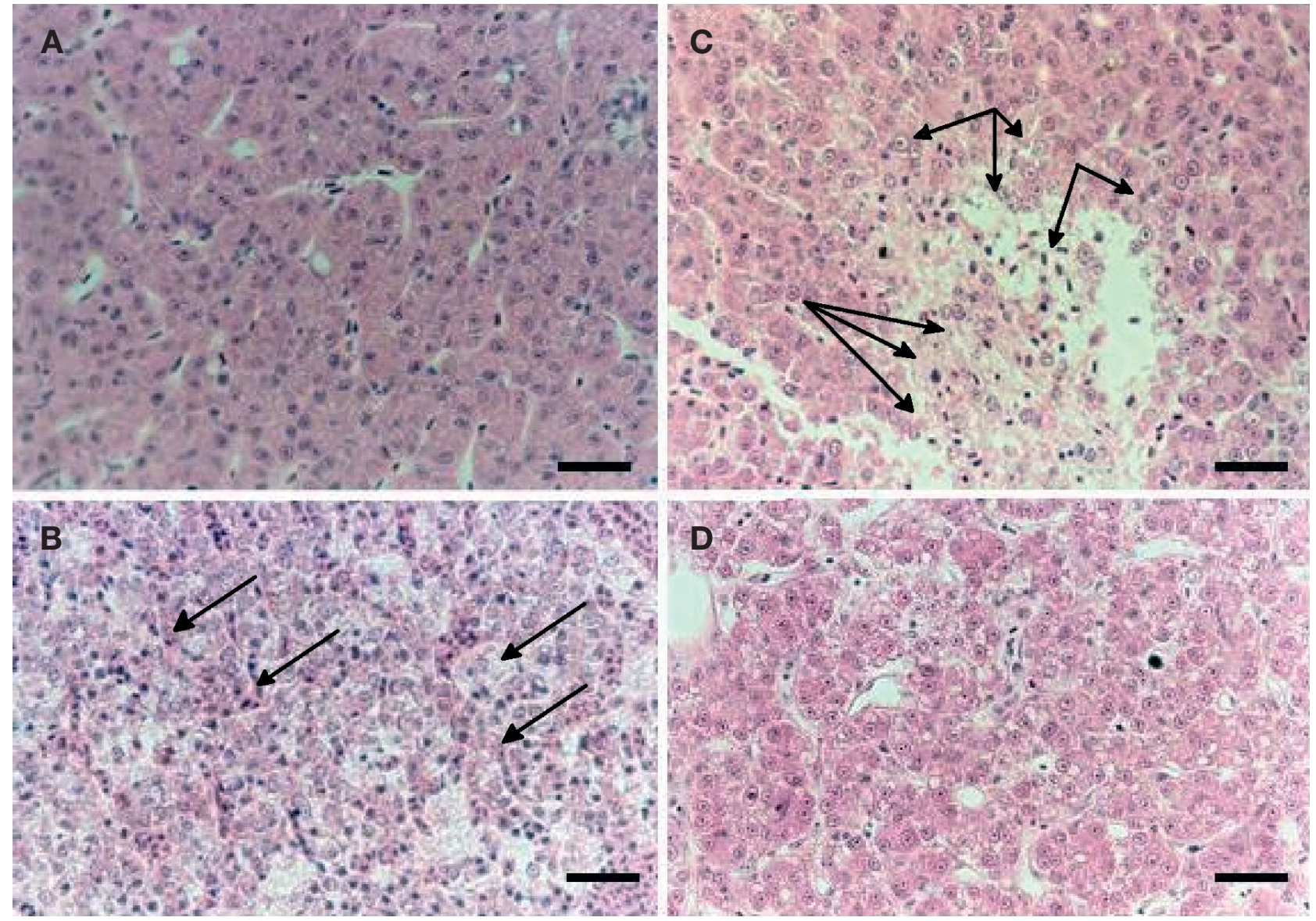

Fig. 5. Oncorhynchus mykiss. Main lesions observed in liver from fish experimentally infected. (A) Liver from control fish at Day 8 p.i. (B) Partial loss of hepatic cell-cord structure associated with extensive haemorrhages in the liver (arrows) from fish infected with rIHNV at Day 4 p.i. (C) Necrotic areas in which the nuclei showed marked nucleoli and condensed chromatin (arrows) from fish infected with rIHNV at Day 4 p.i. (D) High prevalence of cytoplasmatic vacuolization in hepatic cells of fish infected with rIHNV-Gvhsv at Day 4 p.i. Hematoxylin and eosin stain. Scale bars $=20 \mu \mathrm{m}$

the hepatic cell-cord structure, generalised haemorrhages, vacuolized cytoplasm and karyorrhectic nuclei (Fig. 5B). The cellular necrotic areas were associated with haemorrhages (Fig. 5C). A decrease in lesions intensity was observed at Day 14 p.i. Lesions produced by rIHNV in the kidney and liver showed a slightly higher intensity than those caused by wtIHNV, whereas the rIHNV-Gvhsv produced less intense damage. At Day 8 p.i. high prevalence of cytoplasmatic vacuolization or karyorrhectic nuclei in hepatic cells (Fig. 5D) were observed in liver from fish infected with rIHNV-Gvhsv.

Interestingly, and in accordance with the mortality results, rIHNV-Gmut induced the same intensity of lesions in the kidney and liver as those caused by wtIHNV. By contrast, rIHNVmut-Gmut did not cause any important lesions in the examined organs (Fig. 6).

Other organs, such as spleen, brain and muscle were always less affected. The wtIHNV caused serious lesions in the spleen (splenocyte necrosis with karyorrhectic nuclei and disappearance of ellipsoids), while only slight changes such as small haemorrhages and more erythrocytes present in blood vessels than in controls were observed in the brain (Fig. 7B, C). In muscle of fish infected with wtIHNV, the muscular fibres lacked striation, showing splitting along with cellular debris and presence of erythrocytes between the fibres (Fig. 7F,G). Necrotic areas, haemorrhages and clusters of erythrocytes were not numerous (Fig. 7G). rIHNV and rIHNV-Gvhsv induced slight changes resembling those caused by wtIHNV in muscle and spleen, though less intense. Neither rIHNV-Gmut nor rIHNVmutGmut caused any serious lesions in the muscle; however haemorrhages, and occasionally hypertrophied fibres, were observed at Days 8 and 14 p.i. (Fig. $7 \mathrm{G}, \mathrm{H}$ ). No lesions were observed in the brain of fish infected with rIHNV-Gvhsv, rIHNV-Gmut and rIHNVmutGmut. 

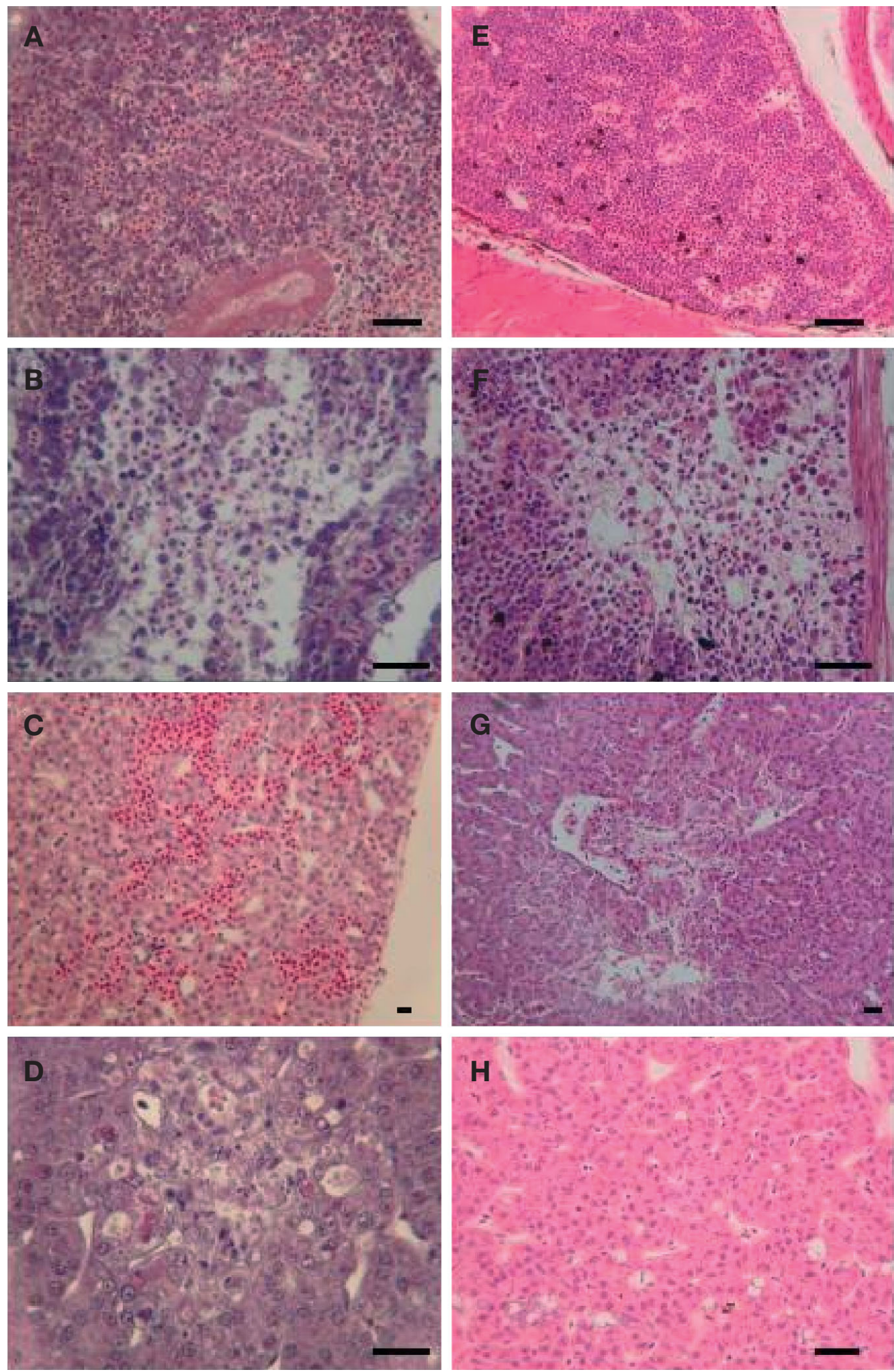

Fig. 6. Oncorhynchus mykiss. Histological sections of tissue infected with (A-D) rIHNV-Gmut and (E-H) rIHNVmut-Gmut. rIHNV-Gmut induced lesions during all days sampled, mainly in $(\mathrm{A}, \mathrm{B})$ kidney (sections showing necrosis of the hematopoietic tissue) and $(C, D)$ liver (sections showing important haemorrhages, disappearance of hepatic cord structure and necrosis). The rIHNVmut-Gmut only induced lesions at the onset of the disease (Day 4 p.i.) in (E,F) kidney (sections showing haemorrhages and necrosis of the hematopoietic tissue) and (G) liver (sections showing haemorrhages generalized, disappearance of hepatic cord structure and necrosis). (H) shows the liver after $8 \mathrm{~d}$ p.i. with no observed lesions. Hematoxylin and eosin stain. Scale bars $=20 \mu \mathrm{m}$ 

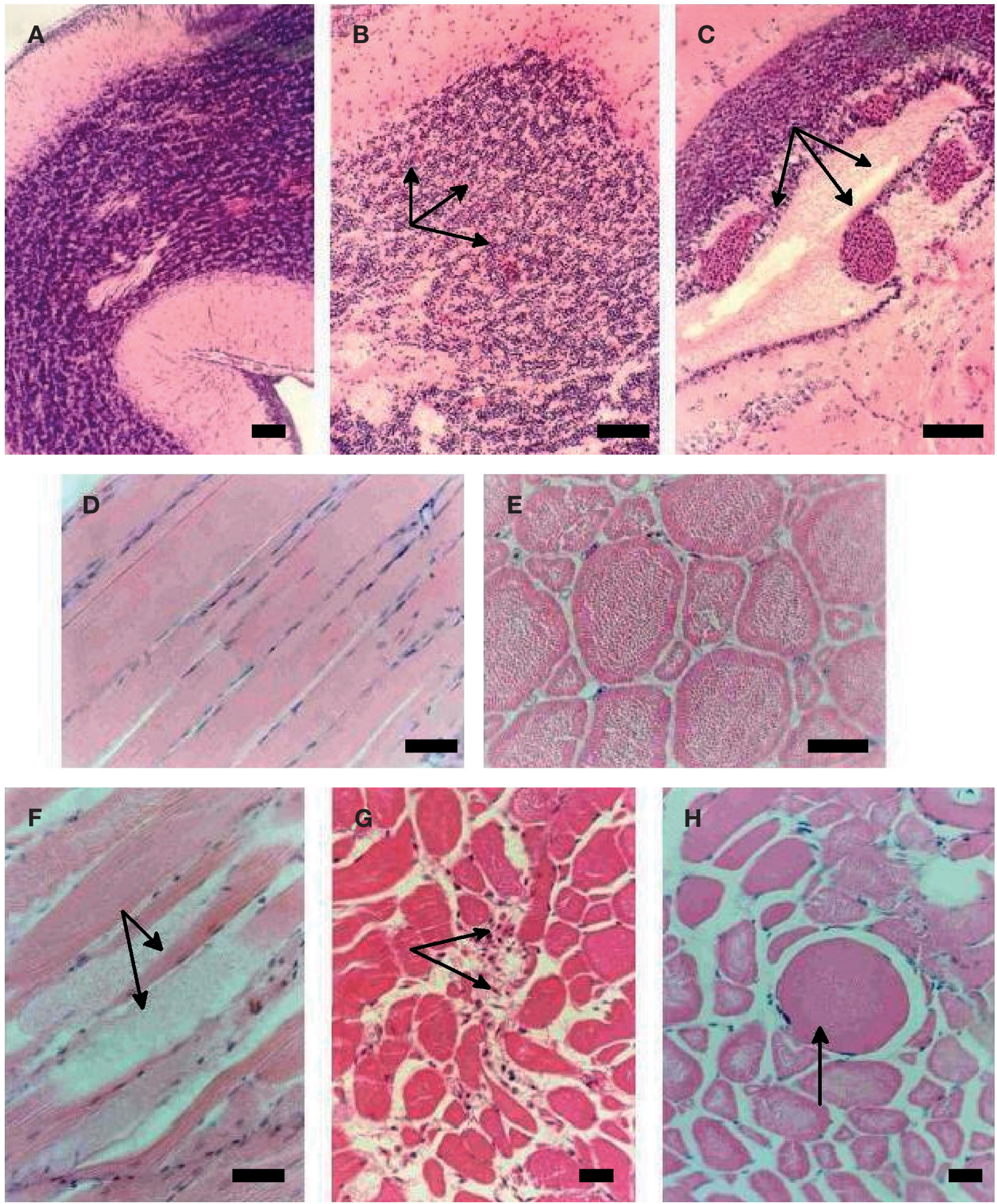

Fig. 7. Oncorhynchus mykiss. Main lesions observed in the brain and the muscle from experimentally infected fish. (A) Brain from a control fish. (B,C) Brain from fish treated with IHNV wild type showing (B) light haemorrhages, and (C) erythrocyte increase in blood vessels. (D) Longitudinal and (E) transverse sections of muscle from control fish. (F-H) Muscle from wild type IHNV showing $(\mathrm{F})$ necrotic areas and $(\mathrm{G})$ haemorrhages and clusters of erythrocytes between the fibres. Occasionally, as in (H), hypertrophied fibres appeared in fish infected with rIHNVmut-Gmut. Hematoxylin and eosin stain. Scale bars $=20 \mu \mathrm{m}$ 


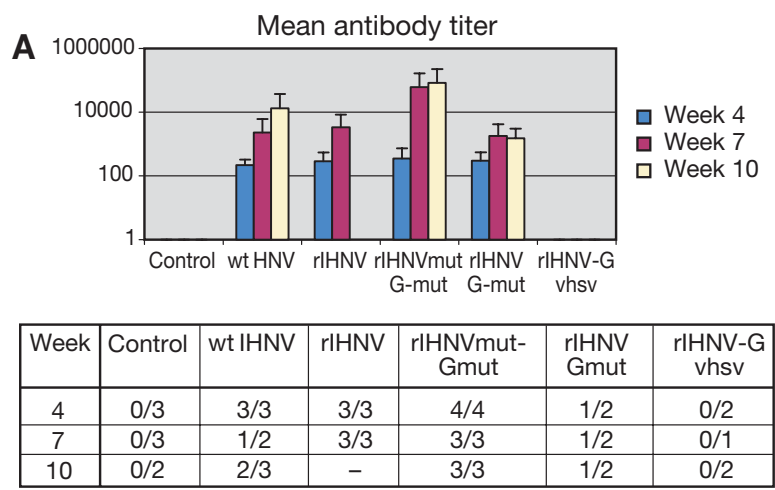

\section{B}

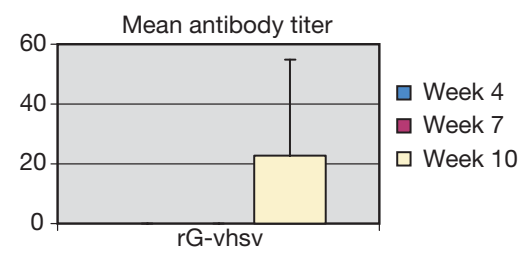

\begin{tabular}{|c|c|}
\hline Week & $\begin{array}{c}\text { rlHNV-G } \\
\text { vhsv }\end{array}$ \\
\hline 4 & $0 / 2$ \\
7 & $0 / 1$ \\
10 & $1 / 2$ \\
\hline
\end{tabular}

Fig. 8. Oncorhynchus mykiss. Mean antibody titer against (A) IHNVs and (B) VHSV from fish experimentally infected with the recombinant viruses. Data in histograms are expressed as mean antibody titer $+\mathrm{SD}$. Data in tables represent: Number of responsive fish / Total number of fish analysed

\section{Viral replication}

All the viruses studied here were tested for their ability to replicate in different organs. Using the immunoperoxidase assay, wtIHNV, rIHNV, rIHNV-Gvhsv, rIHNV-Gmut and rIHNVmut-Gmut were detected at Day 7 p.i. in the spleen and the kidney. At Day 15 p.i., rIHNV was detected in the kidney and the spleen, while rIHNV-Gvhsv, rIHNV-Gmut and rIHNVmutGmut were only detected in the kidney. Neither wtIHNV nor any recombinant were detected in any of the brain homogenates. No virus was detected in control fish throughout the study.

To further quantify the replication of each recombinant virus in the different tissues, a semi-nested RT$\mathrm{PCR}$, a more sensitive technique for the detection of IHNV replication, was performed. Using this method, wtIHNV, rIHNV and rIHNV-Gmut genomes were detected in all the organs (kidney, liver, spleen and gills), as early as $1 \mathrm{~d}$ p.i. In contrast, rIHNVmut-Gmut genome was detected in the kidney of all the fish sampled at Day 1 p.i., but was detected in the kidney and gills of only one fish at Day 3. No viral amplification was ever obtained in control fish.

Through 2 distinct methods, these data provide evidence that all the studied viruses (wtIHNV, rIHNV, rIHNV-Gvhsv and rIHNV-Gmut, but also rIHNVmutGmut) efficiently replicate in all the organs tested.

\section{Antibody production}

The finding that all assayed recombinant IHNVs infected and replicated in rainbow trout, but induced different degrees of pathogenicity, prompted us to test their ability to induce antibody production in trout in response to virus infection. Antibody production in trout in response to rIHNV, rIHNV-Gvhsv, rIHNVGmut and rIHNVmut-Gmut infection was analyzed and compared with wtIHNV. Fish infected with the wtIHNV and recombinant viruses rIHNV, rIHNVGmut and rIHNVmut-Gmut induced antibodies against IHNVs that increased in titers with time from Week 4 to Week 10 (Fig. 8A). The highest antibody production was observed in response to rIHNVmut-Gmut. In contrast, fish infected with rIHNV-Gvhsv did not produce quantities of IHNV antibodies that could be detected through this ELISA methodology.

VHSV antibodies were detected in fish infected with rIHNV-Gvhsv, although the number of responsive fish and the titer were lower than for wtIHNV (Fig. 8B). No IHNV or VHSV antibodies were ever detected in control fish.

\section{DISCUSSION}

In the present study, we show that different recombinant IHNVs generated by reverse genetics (rIHNV, rIHNV-G-vhsv, rIHNV-Gmut and rIHNVmut-Gmut) exhibited different levels of virulence, as assessed by mortality rates and histopathology, in the experimental infections conducted by bath immersion and intraperitoneal injection. For wtIHNV, our results agree with previous reports of experimental infections by other authors (Yasutake \& Amend 1972, Yamamoto \& Clermont 1990, Drolet et al. 1994), showing that the mortality rapidly increased, peaking at Day 5 p.i. The experimental infections conducted with all the different recombinant IHNV viruses, except for rIHNVmutGmut, exhibited a similar mortality pattern. By immersion, the mortality induced by the rIHNVmut-Gmut was delayed $2 \mathrm{~d}$ in comparison with that caused by the rIHNV-Gmut, and the rate at the end of the experiment was much lower in the rIHNVmut-Gmut than in the rIHNV-Gmut. This decrease may be a result of a slight attenuation of the virus virulence. Thus, and this was unexpected, our data demonstrate that the introduction of 2 mutations in amino acid positions 78 and 218 of the G gene is not sufficient to decrease the rIHNVGmut virulence. This result was unexpected with regard to conclusions of Kim et al. (1994) on studies of IHNV neutralization-resistant variants exhibiting different degrees of attenuation (see later in 'Discussion'). However, we showed that additional mutations located 
in the $\mathrm{N}$, the $\mathrm{P}$ and the $\mathrm{M}$ genes (rIHNVmut-Gmut) did efficiently reduce rIHNV-Gmut virulence.

Interestingly, for the recombinant viruses studied here (rIHNV, rIHNV-Gvhsv and rIHNV-Gmut), it was found that infected moribund fish developed typical external symptoms of wtIHNV infection (Amend et al. 1969, Wolf 1988, Bootland \& Leong 1999, Smail 1999). It is noticeable that these symptoms (dark coloration, distended visceral cavity, exophthalmia and petechial haemorrhages at the base of the fins and abdomen, and globally pale internal organs, probably due to anemia) were also observed in fish infected with rIHNVGmut. No external symptoms were observed in fish infected with rIHNVmut-Gmut. These results are in accordance with the study of fish infected with the RB-1 attenuated IHNV neutralization-resistant variant (Kim et al. 1994). Histopathologically, the lesions observed in fish infected with wtIHNV and the recombinant viruses were also similar to those previously described for IHNV infections (Amend et al. 1969, Yasutake \& Amend 1972, Wolf 1988). Some differences in the intensity of the lesions were observed. The recombinant viruses mainly targeted the liver and the hematopoietic tissue of the kidney and the spleen, as already described for wtIHNV (Yasutake \& Amend 1972). The histological study conducted on rIHNVGmut infected fish also showed the presence of lesions, associated with a high rate of mortality. rIHNV-Gmut viral infection was able to induce histological lesions with clinical signs and mortality. However, the RB-1 escape mutant, generated by MAb selection pressure, was strongly attenuated (Kim et al. 1994). The authors correlated this apathogenic phenotype with the presence of 2 mutations in positions 78 and 218 of the G protein. A modification of the viral tropism was also described: RB-1 mutant antigens were mainly detected in the brain and only weakly in liver, opposite to the distribution pattern observed for wtIHNV. Interestingly, this did not occur in the recombinants used in our study where both rIHNV-Gmut and rIHNVmutGmut mutants, sharing mutations with RB-1 in the G gene, did not induce lesions in the brain, and genome replication of both viruses was never detected by immunochemistry or by RT-PCR in the brain. In contrast, both rIHNVmut-Gmut and rIHNV-Gmut mutants induced lesions in the liver, where the viruses were always detected. It should be noted that the RB-1 strain that Kim et al. (1994) described as having a decreased pathogenicity and tropism was analysed only for the $\mathrm{G}$ protein, and no other IHNV gene was analysed or sequenced. It could be the case that the RB-1 strain not only had the 2 mutations in $\mathrm{G}$, but could also have had other changes in the viral genome. Thus, the molecular basis of RB-1 strain attenuation described by Kim et al. (1994) should be further investigated.
It has been previously shown that in IHNV, the heterologous exchange of glycoprotein genes is possible (Biacchesi et al. 2002) as illustrated by the generation by reverse genetics of an IHNV in which the IHNV G gene was replaced with the VHSV G gene (rIHNVGvhsv) (Biacchesi et al. 2002). This virus was already shown to efficiently replicate in cell culture, and to be virulent for rainbow trout experimentally infected by bath. In this latter study, rIHNV-Gvhsv was recovered from kidneys, spleens and, in one case, the brain of infected fish. Interestingly, it was found here that replacement of the G protein by VHSV G protein (rIHNV-Gvhsv) did not modify the tropism or the histological lesions induced by the viral infection. Although it was previously observed that rIHNV-Gvhsv induced plaques with a size between that of the plaques induced by IHNV and VHSV in cell culture (Biacchesi et al. 2002), we show here that histological lesions induced by this recombinant in experimental infections did not show intermediate characteristics between wtIHNV and VHSV. Moreover, all the characteristics of rIHNV-Gvhsv described in the present study were very similar to those obtained in experimental infections with wtIHNV, except for antibody production in infected fish.

Several authors have investigated the route of entry and progression of IHNV (Yamamoto 1990, Yamamoto \& Clermont 1990, Drolet et al. 1994) suggesting that IHNV infection progressed from gills into the circulatory system and from the oral region into the gastrointestinal tract and then into the circulatory system. In the present work, the presence of IHNV wild type and rIHNV genomes were detected by RT-PCR as early as $1 \mathrm{~d}$ p.i. in the gills. Interestingly, a similar progression of viral infection was observed at the first steps of infection for fish infected with rIHNVmut-Gmut and rIHNV-Gmut as well.

LaPatra et al. (1993) and Lorenzen \& LaPatra (1999) showed that rainbow trout can produce specific and highly functional antibodies that are able to neutralise IHNV pathogenicity in vitro as well as in vivo with an increase in antibody production with time. The kinetics and antibody production following an experimental infection with the recombinant viruses were similar to those previously described for IHNV. rIHNV-Gvhsv, expressing the VHSV G protein, was able to induce specific VHSV antibodies but not IHNV antibodies. As expected, rIHNV-Gmut was able to stimulate efficiently IHNV antibody production that increased up to a peak in the tenth week. Interestingly, antibody production was higher than that induced by rIHNV or IHNV wild type. This could explain the delay in the progression of the disease and the lower intensity of the lesions observed in fish treated with rIHNVmutGmut. 
Finally, the present study demonstrates that rIHNVmut-Gmut is probably the most interesting candidate for the development of a suitable vaccine, since it induced low levels of lesions in comparison with the other assayed viruses in both experimental infections by bath or by intraperitoneal injection, and the antibody production was the highest of all viruses used.

Acknowledgements. This work was supported by the projects FAIRCT 98-4398 (European Union) and BIO 2000-0906 (Spanish Ministerio de Ciencia y Tecnología). A.R. acknowledges the CSIC for the I3P fellowship. We thank J. Coll (INIA, Spain) for providing the 1G7 antibody and B. Villaverde and J. R. Caldas for their technical assistance and co-operation. M. LeBerre (INRA, France) is fully acknowledged for virus production.

\section{LITERATURE CITED}

Amend DF, Yasutake WT, Mead RW (1969) A haematopoietic virus disease of rainbow trout and sockeye salmon. Trans Am Fish Soc 98:796-804

Aranguren R, Tafalla C, Novoa B, Figueras A (2002) Experimental transmission of encephalopathy and retinopathy induced by nodavirus to sea bream, Sparus aurata L., using different infection models. J Fish Dis 25:317-324

Biacchesi S, Thoulouze MI, Béarzotti M, Yu YX, Brémont M (2000) Recovery of NV Knockout Infectious Hematopoietic Necrosis Virus expressing foreign genes. J Virol 74: 11247-11253

Biacchesi S, Yu YX, Béarzotti M, Tafalla C, Fernandez-Alonso M, Bremont, M (2000) Rescue of synthetic salmonid rhabdovirus minigenomes. J Gen Virol 81:1941-1945

Biacchesi S, Béarzotti M, Bouguyon E, Brémont M (2002) Heterologous exchanges of the glycoprotein and the matrix protein in a Novirhabdovirus. J Virol 76:2881-2889

Bootland LM, Leong JC (1999) Infectious haematopoietic necrosis virus. In: Woo PTK, Bruno DW (eds) Fish diseases and disorders, Vol 3: viral, bacterial and fungal infections. CAB International Publishing, New York, p 57-121

Drolet BS, Rohovec JS, Leong JC (1994) The route of entry and progression of infectious hematopoietic necrosis virus in Oncorhynchus mykiss (Walbaum): a sequential immunohistochemic study. J Fish Dis 17:337-347

Engelking HM, Leong JC (1989a) Glycoprotein from infectious hematopoietic necrosis nirus (IHNV) induces protective immunity against five IHNV types. J Aquat Anim Health 1:291-300

Engelking HM, Leong JC (1989b) The glycoprotein of infectious hematopoietic necrosis virus elicits neutralizing antibody and protective responses. Virus Res 13:213-230

Huang C, Chien MS, Landolt M, Batts W, Winton J (1996) Mapping the neutralizing epitopes on the glycoprotein of infectious haematopoietic necrosis virus, a fish rhabdovirus. J Gen Virol 77:3033-3040

Ito $M$, Takayama M, Yamada $\mathrm{K}$, Sugiyama M, Minamoto N (2001) Rescue of rabies virus from cloned cDNA and identification of the pathogenicity-related gene: glycoprotein gene is associated with virulence for adult mice. J Virol 7: 9121-9128

Johnson MC, Simon BE, Kim C, Leong JC (2000) Production of recombinant snakehead rhabdovirus: the NV protein is not required for viral replication. J Virol 74:2343-2350
Kim CH, Winton JR, Leong J (1994) Neutralization-resistant variants of infectious hematopoietic necrosis virus have altered virulence and tissue tropism. J Virol 68:8447-8453

Kurath G, Leong JC (1985) Characterization of infectious hematopoietic necrosis virus mRNA species reveals a nonvirion rhabdovirus protein. J Virol 53:462-468

LaPatra SE, Turner T, Lauda T, Jones GR, Walker S (1993) Characterization of the humoral response of rainbow trout to infectious hematopoietic necrosis virus. J Aquat Animal Health 5:165-171

Lawson ND, Stillman EA, Whitt MA, Rose JK (1995) Recombinant vesicular stomatitis viruses from DNA. Proc Natl Acad Sci USA 92:4477-4481

Lorenzen N, LaPatra SE (1999) Immunity to rhabdoviruses in rainbow trout: the antibody response. Fish Shellfish Immunol 9:345-360

McAllister PE, Wagner RR (1975) Structural proteins of two salmonid rhabdoviruses. J Virol 15:733-738

Miller TA, Rapp J, Wastlhuber U, Hoffmann RW, Enzmann PJ (1998) Rapid and sensitive reverse transcriptasepolymerase chain reaction based detection and differential diagnosis of fish pathogenic rhabdoviruses in organ samples and cultured cells. Dis Aquat Org 34:13-20

Sánchez C, Coll J, Domínguez J (1991) One-step purification of the major rainbow trout immunoglobulin. Vet Immunol Immunopathol 27:383-392

Schnell MJ, Mebatsion T, Conzelmann KK (1994) Infectious rabies viruses from cloned cDNA. EMBO J 13:4195-4203

Schütze H, Enzmann PJ, Kuchling R, Mundt E, Niemann H, Mettenleiter TC (1995) Complete genomic sequence of the fish rhabdovirus infectious haematopoietic necrosis virus. J Gen Virol 76:2519-2527

Schütze H, Mundt E, Mettenleiter TC (1999) Complete genomic sequence of viral hemorrhagic septicemia virus, a fish rhabdovirus. Virus Genes 19:59-65

Shapiro DA, Adkison MA, Kaattari SL (1997) Antibody affinity analysis using the ELISA. In: Lefkovits I (ed) Immunological methods manual, Vol 4. Academic Press, San Diego, p 2354-2364

Smail DA (1999) Viral haemorrhagic septicemia. In: Woo PTK, Bruno DW (eds) Fish diseases and disorders, Vol 3: Viral, bacterial and fungal infections. CAB International Publishing, New York, p 123-147

Thoulouze MI, Bouguyon E, Carpentier C, Brémont M (2004). Essential role of the NV protein of Novirhabdovirus for pathogenicity in rainbow trout. J Virol 78:4098-4107

Tomasec J, Fijan N (1971) Virusne balesti riba (viral disease of fish). Final report on research under a part of project, 6n/ 1966, Zagreb

Whelan SP, Ball AL, Barr JN, Wertz GT (1995) Efficient recovery of infectious vesicular stomatitis virus entirely from cDNA clones. Proc Natl Acad Sci USA 92:8388-8392

Wolf K (1988) Infectious hematopoietic necrosis. In: Wolf K (ed) Fish viruses and fish viral diseases. Cornell University Press, Ithaca, NY, p 83-114

Yamamoto T (1990) Multiplication of Infectious Hematopoietic Necrosis Virus in rainbow trout following immersion infection: Whole-body assay and immunohistochemistry. $\mathrm{J}$ Aquat Anim Health 2:271-280

Yamamoto T, Clermont TJ (1990) Multiplication of Infectious Hematopoietic Necrosis Virus in rainbow trout following immersion infection: Organ assay and electron microscopy. J Aquat Anim Health 2:261-270

Yasutake WT, Amend WF (1972) Some aspects of pathogenesis of infectious hematopoietic necrosis (IHNV). J Fish Biol $4: 261-264$

Submitted: June 28, 2004; Accepted: September 2, 2005

Proofs received from author(s): December 12, 2005 